\title{
Using RFID's for Job-Site Productivity Evaluation of Labor and Crews
}

\author{
Edgar P. Small ${ }^{a *}$ \\ ${ }^{a}$ University of Delaware, Department of Civil \& Environmental Engineering, Newark, DE 19716 USA
}

\begin{abstract}
Radio frequency identification (RFID) provide unique identification of objects through non-contact scanning. RFID technology has developed to the point where the price point has made the technology pervasive for inventory control and management of commercial products. Inventory control and storage management is paramount within construction and RFID's provide a useful alternative to bar-codes and other inventory management approaches. RFID's have the additional benefit of providing short-range communication capabilities which could enhance security and theft prevention through 'geofencing' style control. Material and equipment management are natural applications for the technology. The question naturally arises as to the impact of RFID on labor management and how such technology could be used to improve job-site productivity. This has been explored through research performed on-site in the Middle East to track location of workers and to post-process information for productivity assessment. The results of the study will be presented, the advantages and disadvantages discussed, and future research directions proposed.
\end{abstract}

(C) 2018 The Authors. Published by Diamond Congress Ltd., Budapest University of Technology and Economics Peer-review under responsibility of the scientific committee of the Creative Construction Conference 2018.

Keywords: construction; productivity; RFID

\section{Introduction}

Proficiency within a wide variety of tasks is required in order to deliver a construction project successfully: on-time, within budget, at the quality prescribe and in a safe manner. The construction management team must determine and define the methods and procedures necessary to assemble the components and to complete the project. The materials and/or components must be acquired and delivered to the installation location. Labor and equipment must be assembled and must be efficient and effective at performing the tasks required for installation. If the materials do not show up at the proper time, labor and equipment are unable to install and both costs and time increase. Likewise, if labor and equipment are not effective at installing or have diminished productivity, costs and time increase. These situations jeopardize the successful installation of a given item. If too many items have delays, the economic success of the project may be jeopardized and the project completion dates delayed. To avert this and ensure project success, the construction management team must ensure that the materials, labor and equipment are managed effectively.

Starting with the materials, it is easy to see that the construction management team must know where the materials are and must coordinate effectively to ensure that they get to the right place on the job-site at the right time. Coordinating the movement of the materials through the supply chain and/or on the job-site is the central purpose of a materials management system. Without an effective and proactive materials management approach, it would be difficult to ensure success in construction project delivery. The systems which have been used historically were heavily reliant upon paper-based tracking and reporting, which has now evolved to the use of bar-codes, RFID's and proactive notifications. 
Materials management and product supply-chain management are prototypical applications for RFID implementation. With RFID's, items can be tracked and traced as they progress through the supply chain to the consumer or point of installation. RFID's have also been proven to be effective for equipment management. This includes both large-scale equipment, such as backhoes, graders, etc., and small tools. For both materials and equipment, RFID's are proven technologies for tracking and for inventory management.

Of interest is the use of RFID's for productivity and for development of a system which provides support for project managers to identify and correct productivity issues early. This is a bit easy to see when looking at equipment management for large scale machinery which operates on a cycle. Placing RFID readers at points through the cycle can be a useful tool for productivity assessment of the process to determine times for loading, hauling, unloading and returning. Where deviations occur and are identified, corrective action can be taken efficiently and effectively. Small tools and hand-held power equipment are generally not used and evaluated on the basis of a cycle. For these tools, RFID's provide effective management information in terms of tracking, locating and associating the equipment with project personnel and/or job-site location.

For both the materials management and the equipment management applications, RFID's also provide information that can have positive impact on security and theft control, especially where controlled access to the site is possible. While productivity applications may be lacking, opportunities for use of RFID's can been seen and creative applications can readily be conceptualized if challenging the status quo.

Labor management on a construction site can also benefit through the use of RFID's. Employees can be 'clockedin' when entering the job-site and their location on site partially determined, depending on the density of RFID readers positioned on site. This can replace more expensive finger-print sensors and increase the reliability of the information collected and maintained since it is not dependent on the action of the employee. It is also a more effective way to ensure proper access control in addition to tracking and employee management.

Where employees are working on cyclical tasks, such as delivering materials, labor productivity can be ascertained by tracking employees progress and timings through the cycle. This is also applicable for equipment operators, though that information becomes somewhat redundant with the equipment cycle data. The sensor, however, have no ability to recognized the tasks that are being performed at a given time. They simply recognize the location and transfer information that can then be acted upon using an if-this-then-that type of approach.

The question naturally arises as to whether the RFID systems can provide further information that would be useful for productivity assessment. This question was explored in by the authors through a small research project initiated in the Middle East with the intent of demonstrating proof of concept for wider implementation. The results and the background work performed to investigate the feasibility of the approach are presented herein. The paper begins with a definition of RFID's and a discussion of their uses and implementation within the marketplace. The goal is to explore the use of the technology as a tool for labor productivity and research efforts focusing on the use RFID's for productivity is discussed. This includes discussions of research for materials management, equipment management and labor management with the objective of culling useful information for productivity evaluation. The research discussion is followed by a summary of experiences with implementation of RFID on a high-rise commercial job-site in the Middle East. Advantages and limitations of the intended research activity is identified and unexpected difficulties with the labor workforce are discussed. The goal of the work was to provide proof of concept in order to extend and expand the research and a plan for this area is outlined prior to the discussion of conclusions.

\section{What are RFID's, Where Are They Used, and How is the Technology Employed?}

RFID stands for radio-frequency identification, which has become increasingly prevalent in commerce throughout the globe. An RFID tag enables storage of electronic information that can be associated with a given object, such as a piece of equipment or a product. Products and materials in construction may be discrete, such as a door or window. These items allowing individual tagging; however, other materials may be 'fluid' and unable to be tagged. Such materials, such as plastic concrete, can still be managed with RFID tags as long as there is a surrogate placement of RFID tags. This could include placement of the tag on a delivery ticket or on the delivery truck.

In most applications, the RFID tags are passive and receive power from the RFID reader when interrogated. Active, battery powered tags are available which provide greater range; however, they are more expensive and physically larger in size to accommodate the power source. Regardless of the tag technique used, RFID's provide the same benefit. Unlike bar-codes, RFID's do not require line of site or visual scanning in order to convey the information. Tags can replace the bar-code entirely; however, in most commercial applications of RFID's, bar codes are retained. The RFID tag provides a complement to the bar code, which provides redundancy and is retained as a back-up system. 
RFID's are used in an extremely wide variety of applications including access management, toll collection, inventory tracking and tracking of items through logistical processes (such as luggage or packages), security of inventory, and timing of sports events. The technology has become ubiquitous within commerce and creative applications are emerging to use RFID's for a wide variety of additional purposes. This includes using RFID technology to:

- personalize advertisements based on smart-cards linked with facial recognition and social media integration,

- locate underground assets as part of an infrastructure management program.

- track, optimize and effectively bill for waste management,

- manage and support maintenance on commercial aircraft

- minimize traffic delays through intelligent transportation systems linked to toll collection systems, and,

- for micro-chipping of pets.

A study on the use of RFID technology specifically within a retail product supply chain identified the following primary applications, some of which are very applicable on a construction site. As identified by Liukkonen [1], these are as follows:

- "Warehouse inventory tracking and management

- i.e. 'Manufacturing logistics'

- Control of material flows

- Management of picking, receiving, and shipping

- Process monitoring, management, and control

- Tracking of WIP and assembly status during production

- Quality control and process management on the job-shop level

- Tool management

- Locating production tools using RFID

- Supply chain management of manufacturing tools and instruments

- Control of manufacturing robots and instruments

- Supply chain management

- Distribution of information about the location of individual products progressing through the chain from manufacturers to selling companies and end-users

- Life cycle management

- As above, but concerns the monitoring and management of entire life cycle." [1]

Construction certainly has supply chain concerns and the RFID's can be used for some of the same purposes employed in retail. In construction, the technology can be used for inventory control, to provide access control, tracking of items through the supply chain, to support billing and progress payments, and other applications. Construction, however, is a very unique industry and the implementation of these common applications is very different from retail applications in terms of the process. Opportunities for advancement specifically in the construction arena must also be considered, which has been explored through publication in the RFID Journal [1]. The article, which explored potential market applications for professionals focusing on the sensors, identified the following example uses for RFID technology in construction:

- To monitor equipment and products on the jobsite and to issue alerts of equipment and/or materials and products are not located in the proper areas. This was done to ensure that productivity and the schedule was controlled not to control theft as the site was in a remote area with little opportunity for theft.

- To ensure that tolls and equipment are tracked properly when checked out from storage and equipment sheds. This ensured effective equipment management.

- For inventory management on the site in order to decrease the amount of waste and loss generated.

- To track progress of delivery of large precast units and other modular construction assets.

- For workforce management by tracking the number of workers on the job and their identities automatically when entering and leaving the site.

- To enhance construction safety by identifying when workers were entering hazardous areas, such as high-stories with fall hazards or underground work. 
A variety of creative uses for personnel tracking have been explored and associated commercial products developed. As an example, RFID can track smart-badges for employees entering buildings. With an RFID access control system in place, the sensors and the resulting information can be used for tracking emergency responses. This is particularly useful in hazard prone areas where earthquakes or fires may call for rapid evacuation. The system, if in place, could be used to ensure whether all employees were evacuated and arrived within assembly areas. Using this approach requires deliberate management of access control, by necessity, would require a holistic system requiring employees and building visitors/occupants to be 'badged' at all times. Such culture is commonplace in large organizations and governmental agencies. This application has significant benefit in the event of an emergency, such as an earthquake or fire. [2]

A variety of RFID-based systems are being marketed for personnel tracking, for hospital patient tracking, and for child care/elder care management [3]. The author has observed the application of such systems through RFID-tagged wristbands which then permit care workers to identify where loved-ones are located within a given facility. Systems observed do not provide specific locations like a GPS receiver but rather identify rooms and areas where the RFIDwristbands were last located. Nevertheless, it provides very useful information for management of a particular program, such as nurseries.

\section{Research in RFID Applications in Construction for Productivity}

In a survey of sensor applications in construction performed by Zhang, Cao and Zhao, approximately 90 recent research studies using sensors and sensor networks for construction safety were identified. Of these, one-third of the studies (29 of 91) identified were conducted using RFID's for construction safety management [4]. Other sensor technologies explored for included wireless sensor networks, vision-based sensing, and ultra-wide band (UWB) systems, wireless LAN's, and select proprietary systems. GPS was not pervasive and only used in 6 of the 91 studies identified. This makes sense as GPS is frequently inaccessible when placed in an indoor environment. Safety management research topics employed RFID included research into various developments of integrated safety management systems and accident forewarning systems [4]. Regardless of the system used, the types of data necessary must further be considered. For instance, in order to determine whether an employee is near a particular hazard and is in danger, a level of precision in locating the employee is required. The RFID's must track the position of the tag/employee instead of just a broadly defined location.

RFID's have the capability to track positions of RFID tags with varying accuracy, depending on the location and the arrangement of the readers in relation to the tags. Research done on using RFID's for locating objects on construction sites showed 3.7-meter positional error [4,5,6]. Better results were obtained in other studies which demonstrated a 1.3-meter average positional error [7]. It was recognized that more accuracy could obtained with the use of more advanced algorithms [8]. In work done by Lee, Lee and Park [9], sub-meter locational accuracy was obtained using RFID's. On construction sites, obtaining this level of precision consistently could be tricky due the under-construction nature of the environment and the potential for interference with signals from site objects. Furthermore, the cost effectiveness of employing RFID readers is questionable when considering the large number that would be required to cover the entire construction site [4]. The use case explored in practice must be carefully designed so that reliable results are obtained with the practicable precision of the sensor network.

In evaluating productivity applications of RFID's papers tend to focus on two different areas: construction equipment [11] and construction materials/inventory management where numerous research activity is performed and case studies abound. Research activities also extend materials management beyond the site where items can be tracked through the supply chain. In these applications, it is possible for project management personnel, if given access to the information, to identify the status of deliveries and to get real-time updates of delivery times. This provides useful information into the decision-making process.

Interesting studies using RFID's for a variety of useful applications were identified in the review of available literature. Studies identified includes using RFID-based systems for inventory management in emergency relief operations [12], to managing shared inventory in steel yards [13], and to track large-scale products with multiple delivery challenges in precast plants [14]. Interesting work has also been performed using drones for material tracking on construction sites [15].

In examining the research, it is clear that there are some limitations with RFID's for productivity evaluation and for safety evaluation. The tag/reader combination can isolate positions and transmit information about the worker; however, it does not give insight into the worker activity. To evaluate the behavior of the workforce, an alternative 
approach must be coupled with RFID's. Zhang, et. al., explored the use of other sensor systems but these approaches only impacted the ability to locate a tag or employee. The systems do not have the capability to determine the activity being performed by the employee, whom may be productive or unproductive at various times throughout the day. An alternative approach is required to capture this information. One of the most promising techniques identified in the literature involves automated or 'smart' imagery and video processing. Significant research is on-going in this area for construction performance monitoring [16]. The images, captured using photographs, time-lapse or video livestream, are processed using algorithms and techniques to recognize both the worker and to evaluate the behavior. The algorithms to facilitate this process are rapidly advancing and imagery based approaches show significant promise. Researchers focusing on development of imagery-based systems recognized that "the future of intelligent construction monitory can rely on multiple types of sensors or sensor networks," including RFID's to, "compensate [for] the disadvantages of visual sensors and provide more types of information" [16]. Benefits for these types an integrated approaches are clear for productivity but can also have significant application throughout the construction enterprise.

\section{The Use of RFID's for Productivity: Exploring the Approach in Commercial Construction}

To explore how RFID's could be used for labor productivity, a test case was devised using RFID's for implementation on a commercial construction site in the Middle East. RFID tags were placed on worker hardhats and readers were implemented within a limited area of an active construction site. The readers permitted tracking of when workers entered the area of interest on the job-site. Only the skilled and unskilled labor on the finishing crew were fitted with RFID tags.

It was recognized that locating a worker does not give an indication of the work being performed. Nevertheless, it was expected that RFID incorporation would provide easily obtained data that provides benefit for productivity evaluation. The research was designed so that benefits obtained on a small scale would provide proof of concept as a value-added technique. A more substantial research project would then follow with the approach employed for a larger segment of the workforce. The ultimate goal would be for eventual implementation across site for all employees. As technology advances, other features could be added moving towards a tool permitting near real-time evaluation. In other words, the objective was to start small, demonstrate effectiveness and subsequently expand until a difference could be made across the site and company.

In this study, the system was designed to get an indication of the time that workers spent in a given area. This would then be correlated to the amount of work performed in the area. The specific area chosen for evaluation was limited by the work being performed and access granted by project management team. Constructed masonry partition walls were completed and the labor and crews considered in the study only worked on finishing of the walls with a cementitious skim coat. There were no known conflicts in schedule between trades and no space limitations. No special considerations were required for implementation of the study other than fitting of tags and readers and access to daily reports.

There were significant limitations with the area permitted for the study. The overall site involved the construction of a large commercial tower and a portion of one floor was made available for the study. The planned work was scheduled for 3 days and there was limited opportunity for changing the level of effort or changing the activities and items performed by the crews.

The work being evaluated only involved a small number of workers from part of a large work-force. It was not practical to fit all construction workers with RFID tags as the time availability was limited and the data was expected to provide proof of concept for further study. Workers were informed of the study was being performed and that RFID tags were being used. The research team did not anticipate any issues with this approach since all workers on this site were accustomed to using fingerprint sensors for access tracking and control. When workers passed by a fingerprint sensor on the job-site they were obliged to check-in, regardless of the trade. This was used for monitoring of and accounting for the workers' time. The finger-print data was not used for productivity assessment and was not accessible by the research team. Nevertheless, it was presumed that the laborers would be accustomed to tracking and that there would not be any issue with implementing the RFID's.

A limited number of RFID readers were employed. The readers tracked when workers entered or exited the study area. Entering or exiting was determined on the basis of the time-stamp and for continuing research efforts, an enhanced system would need to be implemented in order to isolate the direction of the worker travel. The data would show progress under the reader but would not indicate whether the worker was entering or exiting the area of interest. This was inferred by the researchers based on the timings and the daily break schedules. In the future, the sensor network could easily be modified to evaluate entrance versus exit automatically without manual input. 
When implementing the systems, it was expected that work would progress unimpeded by the study. This assumption was not valid. Laborers on the affected crews did not welcome the RFID's and either removed them from the hat, cut and damaged them, or used other workers' hardhats. These workers objected to being tracked and this was an understandable recourse. As such, reliable information was not obtained during the initial day of the study. No problems were experienced with the readers, which were largely ignored. Further complications came from the workforce that were not chosen to be part of the study, whom resented that they were not chosen for a special type of task. The resulting pressure on the crew by their co-workers further exacerbated the difficulty with implementing the tags. Despite discussion with foremen and superintendents, worker distrust persisted through the course of the study and limited information was obtained. The information could thus not be correlated with the work performed.

The resistance by the workforce was unexpected by the research team but not unprecedented. It is understood that early implementation of camera surveillance on construction sites had received resistance from the workforce and may still be a sensitive issue among unionized labor. Since the study, efforts were undertaken by the supervisory staff on the site to ensure that workers would not damage the RFID's tags. This was done independent of the study as the workforce is no longer performing tasks within the study area and RFID readers have been removed. Site personnel indicated that it took about 2 weeks for the labor to progress without care of the RFID tags. It is unknown whether similar problems with labor acceptance will occur in future studies.

For future implementations within this marketplace, care must be taken to avoid the problems resulting from labor resistance to being singled-out and having RFID tags. There are many different ways that this could be addressed. In a large-scale implementation, RFID tags could be provided for all hard-hats either as a specific tag akin to the type used for product theft control. Alternatively, RFID tags could be integrated within hard-hat stickers or within clothing worn by workers, such as safety-vests or coveralls. This will become easier as miniaturization becomes more prevalent within the marketplace.

\section{Summary, Conclusion and Future Directions}

This research study was initiated to develop simple, low-cost approaches for improving productivity in commercial construction in the Middle East. The concept initiated involved installing RFID readers within a controlled area on an active construction job-site and to track RFID tags placed on finishing crew skilled and unskilled laborers. The study was intended to demonstrate viability of the approach through a short-term, low-impact implementation. It was hoped that positive results would then lead to further, more extensive studies over the job-site.

The study began by exploring market-accessible products and past implementations in the Middle East and abroad. Commercial products were available for personnel tracking but low-cost RFID systems available within the marketplace were used for proof of concept. For future study and more extensive research, commercial systems available would have benefit due to the value-added capabilities of the software and the approaches to information management. Research publications were also examined and a number of interesting studies were identified. Studies focused on locational capabilities of the sensors, the use of RFID's for inventory management of materials, supply chain issues and optimization with RFID's, equipment management and productivity, safety analysis and other areas. Few studies were found that were coupling productivity evaluation with RFID's. Notable studies in this area were employing imagery and videography which show significant promise, especially when coupled with sensor systems.

There were unexpected difficulties with the workforce when trying to implement the research. These could not be overcome given the small scale of the research and the limited time window for the research activities. As a result, proof of concept will have to be demonstrated in a different environment. Plans are being formulated to look at RFID within labor training centers established for indoctrination of new employees to the systems employed at the site. The difficulty with this approach is that often it may be difficult to correlate workers receiving training with a reasonable level of productivity. Nevertheless, proof of concept will be easier within a controlled environment and will also help with making employees accustomed to the RFID tags. The researcher team is confident that the approach can be demonstrated as effective within this environment.

It is further recognized that the use of RFID tags is going to have limitations when it comes to productivity assessment. Location can be determined but a sensor-only implementation cannot ascertain whether employees are actively engaged in productive work, supportive tasks or idle. Only macro-level productivity can be obtained by looking at the time in proximity to the work task and the overall quantity completed within a given period of time. This information would provide incremental advancement for evaluation purposes but more data would be desired to closer to real-time feedback. It is expected that coupling of the RFID or other sensor approaches with video and imagery will provide this capability in the future. 


\section{References}

[1] Liukkonen, M, "RFID technology in manufacturing and supply chain,” Intl. J. of Computer Integrated Manufacturing, 201528 (8)

[2] RFID Journal, "How is RFID Being Used in the Construction Industry?", RFID Journal, 06/27/2013, http://rfidjournal.com, accessed 4/29/2018

[3] Zeisel, E., White Paper - Emergency Management and Mustering System, RFID4U, http://rfid4u.com, accessed 4/29/2018

[4] GAO RFID, Inc., RFID Personnel Tracking System, http://gaorfid.com/people-locating-rfid-system/, accessed 4/29/2018

[5] Zhang, M.; Cao, T; and Zhao, X, “Applying Sensor-Based Technology to Improve Construction Safety Management,” Sensors 2017, 17(8), 1841; doi:10.3390/s17081841

[6] Song, J.; Haas, C.T.; Caldas, C.H. "Tracking the location of materials on construction job sites." J Constr Eng. Mang 2006, 132, 911-918.

[7] Gu, Y.Y.; Lo, A.; Niemegeers, I. “A survey of indoor positioning systems for wireless personal networks.” IEEE Commun. Surv. Tut. 2009, $11,13-32$.

[8] Razavi, S.N.; Moselhi, O. "GPS-less indoor construction location sensing." Automat. Constr. 2012, 28, 128-136.

[9] Montaser, A.; Moselhi, O. "RFID indoor location identification for construction projects." Automat. Constr. 2014, 39, $167-179$.

[10] Lee, H.S.; Lee, K.P.; Park, M. "RFID-based real-time locating system for construction safety management.” J. Constr. Eng. Manag. 2012, 26, $366-377$

[11] Šopić, M; Vukomanović, M, "Modeling the productivity of construction machinery by using RFID technology," Proc. $13^{\text {th }}$ International Conference on Organization, Technology and Management in Construction." University of Zagreb, 2017, 701-704.

[12] Ozguven, E.; and Kaan, O., "An RFID-based inventory management framework for emergency relief operations." Transportation Research Part C.: Emerging Technologies, 201557 166-187.

[13] Talavera, H.; Banks, J; Smith, N., and Leopoldo, E., "Enhancing the management of shared inventory in the steel industry using RFID: an alternative to bar codes," Intl. J. of Machine Learning and Cybernetics, 2015 6(5), 733-745

[14] Wang, Z; Hu, H; and Zhou, W., "RFID enabled knowledge-based precast construction supply chain." Computer-Aided Civil and Infrastructure Engineering, 2017 32(6), https://doi.org/10.1111/mice.12254

[15] Hubbard, B, Hubbard, S, Wang, Leasure, Ropp, Lofton and Lin, "Feasibility Study of UAE Use for RFID Material Tracking on Construction Sites," Proc. $51^{\text {st }}$ ASC Annual Intl Conference Proceedings 2015

[16] Yang, J; Park, M; Vela, P; and Golparvar-Fard, M, "Construction performance monitoring via still images, time-lapse photos, and video streams: Now, tomorrow and the future.” Advanced Engineering Informatics, 2015 29(2), https://doi.org/10.1016/j.aei.2015.01.011 